\title{
Suprapubic Incisional Hernias: Is Open Surgery a Standard?
}

\author{
B. $\operatorname{Ramana}^{1,2}$
}

Published online: 15 March 2017

(c) Société Internationale de Chirurgie 2017

In this study [1] of 71 cases of SIH, the authors make their case for an open retromuscular mesh repair with lightweight polyester using full-thickness sutures to anchor it. At first glance, this seems to be unexceptional, save for the impressive numbers in the study of a hernia type that is not as common as the umbilical, for example.

However, I take issue with the attempt to sneak SIH in almost as a separate disease entity or hernia, as well as the attempt to portray open surgery as the standard of care for this problem.

SIH are almost the exact same as other ventral hernias with the differences being:

1. The presence of the pubic bone inferiorly

2. The presence of the bladder and major vessels posteroinferiorly

3. The absence of the posterior rectus sheath

However, this is not dissimilar to other hernias that we place under the umbrella 'ventral hernia.' Take subxiphoid hernias, for example. Even flank hernias or hernias through appendectomy scars have peculiarities by themselves. Should they constitute a separate class each for themselves? I think not. We may call each one a special variant in the ventral hernia class.

In the history of laparoscopic hernia surgery, we are all witness to tremendous change, often at a bewildering pace. From the initial days of the intraperitoneal approaches to

\section{B. Ramana}

rambodoc@gmail.com

1 Department of Comprehensive Hernia Surgery, Medica Superspecialty Hospital, 127 Mukundapur, EM Bypass, Kolkata 700099, West Bengal, India

2 Medica Gamma Hospital, Kolkata 700017, West Bengal, India groin hernia using a cigarette roll of mesh shoved into the inguinal canal to the current trends of endoscopic and robotic extraperitoneal posterior component separation techniques, we have come a long way, and our journey does not seem to be complete. Quite the contrary. When we have accepted the laparoscopic approaches to groin, umbilical and ventral hernias as standardized procedures, what stops us from doing so in SIH?

Yes, there is paucity of data. Which is why it is important to keep an open mind and pursue minimally invasive options in a trial setting, to evaluate the short- as well as the long-term results of these approaches.

The trend in ventral hernia surgery in advanced centers around the world seems to be to extraperitonealize the mesh. In suprapubic hernias (as with other locations), this author has been working on the e-TEP approach along with Belyansky et al. [2], and the initial results (to be published) seem to be promising.

How far this will be used across the world for SIH remains to be seen. It is my opinion that to call open retromuscular repair the standard is still premature. We need to collect more data and await the results.

\section{References}

1. Renard Y, Simonneau AC, de Mestier L, Teuma L, Meffert JL, Palot JP, Kianmanesh R (2017) Standard of open surgical repair of suprapubic incisional hernias. World J Surg. doi:10.1007/s00268017-3892-z

2. Belyansky I, Zahiri HR, Park A (2015) Laparoscopic transversus abdominis release, a novel minimally invasive approach to complex abdominal wall reconstruction. Surg Innov 23(2):134-141 